BMJ Open

Diabetes

Research

\& Care

\section{Genetically engineered pigs manifesting pancreatic agenesis with severe diabetes}

\author{
Masaki Nagaya (D) , ,,2 Koki Hasegawa, ${ }^{3}$ Masahito Watanabe, ${ }^{1,3}$ Kazuaki Nakano, ${ }^{1,3}$ \\ Kazutoshi Okamoto, ${ }^{3}$ Takeshi Yamada, ${ }^{3}$ Ayuko Uchikura, ${ }^{1,3}$ Kenji Osafune, ${ }^{4}$ \\ Harumasa Yokota, ${ }^{5}$ Taiji Nagaoka, ${ }^{5}$ Hitomi Matsunari, ${ }^{1,3}$ Kazuhiro Umeyama, ${ }^{1,3}$ \\ Eiji Kobayashi, ${ }^{6}$ Hiromitsu Nakauchi, ${ }^{7,8}$ Hiroshi Nagashima ${ }^{1,3}$
}

To cite: Nagaya M, Hasegawa K, Watanabe M, et al. Genetically engineered pigs manifesting pancreatic agenesis with severe diabetes. BMJ Open Diab Res Care 2020;8:e001792. doi:10.1136/ bmjdrc-2020-001792

- Supplemental material is published online only. To view, please visit the journal online (http://dx.doi.org/10.1136/ bmjdrc-2020-001792).

MN and KH contributed equally.

Received 22 July 2020

Revised 8 October 2020

Accepted 18 0ctober 2020
Check for updates

(C) Author(s) (or their employer(s)) 2020. Re-use permitted under CC BY-NC. No commercial re-use. See rights and permissions. Published by BMJ.

For numbered affiliations see end of article.

Correspondence to Professor Masaki Nagaya; m2nagaya@gmail.com and Professor Hiroshi Nagashima; hnagas@meiji.ac.jp

\section{ABSTRACT}

Introduction Pancreatic duodenum homeobox $1(P d x 1)$ expression is crucial for pancreatic organogenesis and is a key regulator of insulin gene expression. Hairy and enhancer of split 1 (Hes1) controls tissue morphogenesis by maintaining undifferentiated cells. Hes1 encodes a basic helix loop helix (bHLH) transcriptional repressor and functionally antagonizes positive bHLH genes, such as the endocrine determination gene neurogenin-3. Here, we generated a new pig model for diabetes by genetic engineering $P d x 1$ and Hes1 genes.

Research design and methods A transgenic $(\mathrm{Tg})$ chimera pig with germ cells carrying a construct expressing Hes1 under the control of the $P d x 1$ promoter was used to mate with wild-type gilts to obtain Tg piglets. Results The Tg pigs showed perinatal death; however, this phenotype could be rescued by insulin treatment. The duodenal and splenic lobes of the Tg pigs were slender and did not fully develop, whereas the connective lobe was absent. $\beta$ cells were not detected, even in the adult pancreas, although other endocrine cells were detected, and exocrine cells functioned normally. The pigs showed no irregularities in any organs, except diabetes-associated pathological alterations, such as retinopathy and renal damage.

Conclusion $P d \times 1-H e s 1 \mathrm{Tg}$ pigs were an attractive model for the analysis of pancreatic development and testing of novel treatment strategies for diabetes.

\section{INTRODUCTION}

Complications of diabetes mellitus can lead to irreversible damage in various organs and can even result in death. ${ }^{1}$ To improve physiological glucose metabolism in patients with diabetes, several innovative approaches are under development, including islet transplantation, ${ }^{2}$ generation of transplantable new $\beta$ cells derived from human pluripotent stem cells, ${ }^{34}$ stimulation of endogenous $\beta$ cell proliferation, reprogramming of non- $\beta$ cells to $\beta$-like cells, harvesting islets from genetically engineered animals, and encapsulation technology to protect islet transplants from host immune surveillance. ${ }^{5}$ To validate

\section{Significance of this study}

What is already known about this subject?

- Pancreatic duodenum homeobox $1(P d x 1)$ expression is crucial for pancreatic organogenesis and is a key regulator of insulin gene expression. Hairy and enhancer of split 1 (Hes1) controls tissue morphogenesis by maintaining undifferentiated cells. Hes1 encodes a basic helix loop helix (bHLH) transcriptional repressor that functionally antagonizes positive bHLH genes, such as the endocrine determination gene neurogenin-3. Based on these wellknown considerations, here, we generated a new transgenic $(\mathrm{Tg})$ pig model for diabetes by genetic engineering the $P d x 1$ and Hes1 genes.

What are the new findings?

- The Tg pigs showed perinatal death due to severe diabetes; however, this phenotype could be rescued by insulin treatment. The duodenal and splenic lobes of Tg pigs were morphologically long and narrow, whereas the connective lobe and bridge were absent, indicating pancreatic agenesis.

- $\beta$ cells were not detected, even in the adult pancreas, although other endocrine cells were detected, and exocrine cells functioned normally. The pigs showed no irregularities in any organs, except diabetes-associated pathological alterations, such as retinopathy and renal damage.

How might these results change the focus of research or clinical practice?

- Pdx1-Hes1 Tg pigs showed the induction of a stable diabetic phenotype and diabetes-associated complications relatively early. The $\mathrm{Tg}$ pigs were an attractive model for the analysis of pancreatic development and testing of novel treatment strategies for diabetes.

and optimize novel therapeutics for safe application in humans, researchers need to select the appropriate large animal model. It is preferable that such investigations are conducted on animal species with anatomical 
and physiological similarities to humans. Pigs are omnivorous and likely to be obese as well as dyslipoproteinemic like humans. Thus, they are frequently used in medical research as large experimental animals that can provide findings applicable to humans by extrapolation. ${ }^{6}$ Hence, production of a porcine diabetes research model is likely to contribute substantially to studies on the treatment of and on measures related to complications of human diabetes. Although some engineered pig models have been developed for diabetes research, such models are limited. ${ }^{67}$

During embryogenesis, precursor cells initially proliferate to give rise to sufficient numbers of cells; subsequently, these cells stop proliferating and begin differentiation. Proper timing of the transition from proliferation to differentiation is critical for normal development because premature or delayed transition leads to abnormal cell numbers and tissue morphology. ${ }^{8}$ The pancreas exhibits similar mechanisms through which endocrine and exocrine cells differentiate from common precursor cells. Pancreatic transcription factors are involved in pancreas development and $\beta$ cell differentiation. ${ }^{910}$

The homeodomain-containing transcription factor pancreatic duodenal homeobox factor- $1(P d x 1)$ is a master gene involved in early pancreatic development. ${ }^{11}$ In Pdxl-null mice, the phenotype is entirely consistent with complete pancreatic regression observed after initial bud formation. Inactivating mutations in $P d x 1$ cause pancreatic agenesis. ${ }^{12}$ With growth, $P d x 1$ is mainly expressed in $\beta$ cells of islets. It plays key roles in the regulation of insulin gene expression ${ }^{11} 12$ and the maintenance of mature $\beta$ cell function. ${ }^{13}{ }^{14}$ Data from human embryos have shown that $P d x 1$ expression characterizes the earliest steps of pancreatic endoderm development and is expressed sequentially in emergent buds and multipotent progenitors. ${ }^{15}$ Other $P d x 1$ alterations in human have been identified, ${ }^{16}$ including a mild deficit in exocrine pancreas function alongside permanent neonatal diabetes, presumably due to a hypomorphic mutation. ${ }^{17}$

Developmental processes are regulated positively or negatively by multiple basic helix loop helix (bHLH) genes in Drosophila. ${ }^{18} 19$ Among these bHLH genes, hairy and enhancer of split-1 (Hes-1) is expressed by almost all undifferentiated cells and regulates tissue morphogenesis by maintaining undifferentiated cells. ${ }^{8}$ Hes 1 is controlled by the conserved Notch pathway and is a key regulator both of cell fate ${ }^{20-22}$ and embryonic development. ${ }^{23}$ Mutations in Hes1 induce various defects, including reduced pancreas size and absences of a brain, eyes, and thymus in embryos. ${ }^{8}$ In the pancreas, Hes 1 functions as a general negative regulator of bHLH genes, such as the endocrine determination gene neurogenin-3 (Ngn3), and defects in Notch signaling lead to accelerated pancreatic endocrine differentiation. ${ }^{24}$ Thus, several genes, including $P d x 1$, Hes 1, and Ngn 3, orchestrate pancreatic development and endocrine cell function. However, these mechanisms have not yet been exploited for the development of novel large animal models to study the mechanisms of pancreas development and diabetes.

In this study, we describe the development of a transgenic (Tg) pig strain exhibiting unique characteristics caused by overexpression of Hes1 under the control of the $P d x 1$ gene promoter. Our findings provide important insights into the use of this Tg pig model for studies of the pathophysiology of diabetes.

\section{MATERIALS AND METHODS}

\section{Animal care}

All animals were housed and maintained in accordance with Institutional Animal Care and Use committee (IACUC) guidelines. Pigs were bred under conventional conditions in an air-conditioned room and were observed daily by animal husbandry personnel under the supervision of an attending veterinarian.

\section{Production of Pdx1-Hes1 Tg pigs}

We have previously reported Tg pigs carrying a construct that expresses Hes 1 under the control of the $P d x 1$ gene promoter (figure 1A) using intracytoplasmic sperm injection-mediated gene transfer and somatic cell nuclear transfer. From these Tg pigs, a Tg-chimeric boar producing fertile sperm carrying the Pdx1-Hes1 expressing vector was obtained. ${ }^{25}$ The Tg-chimeric boar were mated with wild-type (WT) gilts to acquire Tg piglets exhibiting pancreatic agenesis owing to overexpression of Pdx1-Hes1. These piglets were evaluated in this study. The copy-number of the transgene in the Tg piglets was confirmed by Southern blot analysis.

\section{Genotyping}

Genomic DNA was extracted from tail biopsy specimens of Tg newborn piglets using a DNeasy Blood and Tissue Kit (Qiagen, Hilden, Germany). Genotyping was performed by PCR using primers for Pdx1-Hes 1 transgene sequences. PCR primers used to amplify Pdx1-Hes1 transgene sequences were as follows: 5 -CAATGATGGCTC-

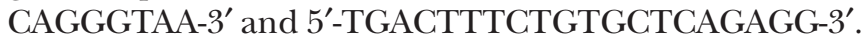
PCR conditions were as follows: $95^{\circ} \mathrm{C}$ for $60 \mathrm{~s}$; followed by 30 cycles of $95^{\circ} \mathrm{C}$ for $30 \mathrm{~s}, 60^{\circ} \mathrm{C}$ for $5 \mathrm{~s}$, and $72^{\circ} \mathrm{C}$ for $60 \mathrm{~s}$.

\section{Estimation of transgene copy-number and integration site}

Genomic DNA was extracted from skin samples of $\mathrm{Tg}$ fetuses using a DNA purification kit (DNeasy Blood and Tissue Kit; Qiagen). The purified genomic DNA ( $5 \mu \mathrm{g})$ was digested with PstI (Takara Bio, Shiga, Japan), separated by gel electrophoresis, and transferred onto a nylon membrane (Hybond N+; GE Healthcare Biosciences, Uppsala, UK). The membranes were blocked for $30 \mathrm{~min}$ at room temperature with blocking reagent (Blocking One; Nacalai Tesque, Kyoto, Japan). After blocking, the membranes were incubated in a hybridization solution (DIG Easy Hyb; Roche Diagnostics, Basel, 

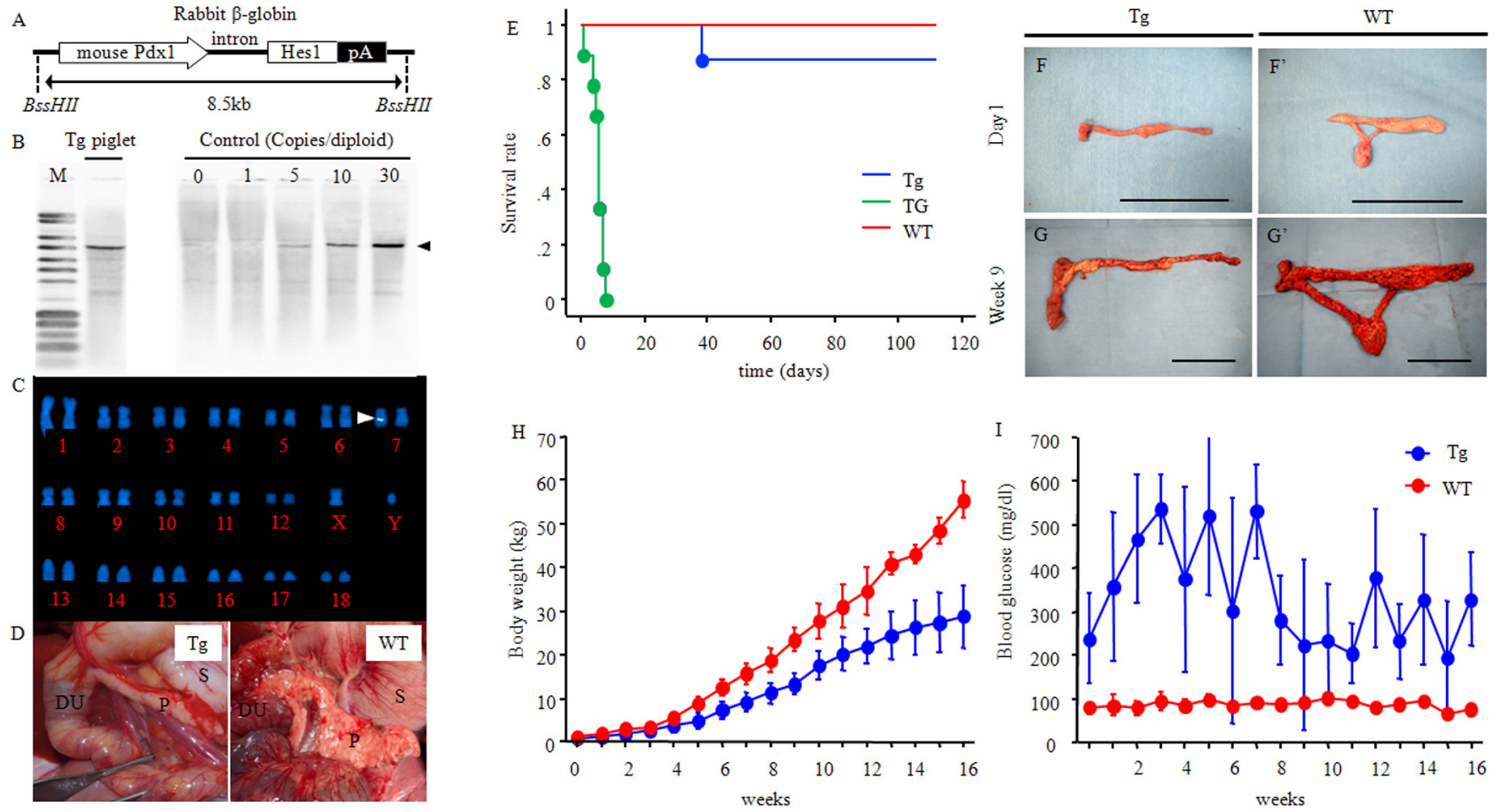

Figure 1 Generation of diabetic Pdx1-Hes1 Tg pigs. (A) Construction of a $P d x 1-H e s 1$ expression vector consisting of the mouse $P d x 1$ promoter, mouse Hes $1 \mathrm{cDNA}$, and rabbit $\beta$-globin $3^{\prime}$ flanking sequence, including the polyadenylation signal (pA). $(\mathrm{B}$ and $\mathrm{C})$ Estimation of transgene copy-numbers and the integration site. (B) Southern blot analysis of Pstl-digested genomic DNA from Pdx1-Hes1 Tg pigs. Offspring of the Tg-chimeric boar carried 15-20 copies of the transgene. The number of transgene copies integrated into the porcine genome was determined by comparison of the hybridization signal with that of the copy-number control. There were 1-30 copies per diploid genome. (C) Chromosomal analysis. Fetal fibroblasts derived from a Tg pig were analyzed by fluorescence in situ hybridization. Integration of the Pdx1-Hes1 transgenes was detected (arrow) in chromosome 7q21 as single-site multicopy transgenes. (D) Pancreatic agenesis in Tg pigs. At 5 weeks, the duodenal and splenic lobes of the pancreas in Tg pigs were morphologically slender and did not fully develop; the connective lobe and bridge were not present (left panel: Tg; right panel: WT). Physiological features. (E) Survival curve. Green line: Pdx1-Hes1 Tg pigs without insulin therapy (TG: $n=9)$. Blue line: Tg pigs with insulin therapy (Tg: $n=9)$. Red line: wild-type pigs (WT: $n=5)$. (F, F', G and $\left.G^{\prime}\right)$ Developmental changes in the whole pancreas. One day ( $F$ and F') and 9 weeks ( $G$ and $\left.G^{\prime}\right)$ after birth are shown. Scale bars: B, B' and C, C': $5 \mathrm{~cm}$. (H) Body weights at specified times. Blue line: Tg, n=4-8. Red line: WT: $n=2-4$. (I) Blood glucose levels. ( $D$ and E) Tg: $n=4-8 ; W T: n=2-4$. Time after birth is indicated as days and weeks. Quantitative data are presented as means \pm SDs. DU, duodenum; Hes1, hairy and enhancer of split 1; M, pUC mix marker; P, pancreas; $P d x 1$, pancreatic duodenum homeobox 1; S, stomach; Tg, transgenic pigs; WT, wild-type pigs.

Switzerland) and hybridized with a digoxigenin (DIG)labeled Pdx1-Hes1 probe prepared by PCR using a DNA labeling reagent (DIG DNA Labeling Mix; Roche Diagnostics). The signals were developed and detected using conventional 5-bromo-4-chloro-3-indolyl phosphate-nitro blue tetrazolium chromogenic stain. The number of transgene copies integrated into the porcine genome was determined by comparison of the hybridization signal with that of the copy-number control, which was diluted to make a standard series (1-30 copies per diploid genome). For chromosomal analysis, fetal fibroblasts derived from a $\mathrm{Tg}$ pig with pancreatic agenesis were cultured and outsourced for fluorescence in situ hybridization (FISH) analysis (Chromosome Science Labo Inc, Sapporo, Japan).

\section{Glucose control}

Pdx1-Hes1 Tg pigs required lifelong administration of exogenous insulin. In humans, the magnitude of disruption of metabolic control by diabetes has been reported, even in young infants and neonates. ${ }^{26}$ However, studies are often limited by difficulties in obtaining control data. To minimize the risk of hypoglycemia and dead in bed syndrome, ${ }^{27}$ a minimum dose of insulin is given by the injection of short-acting (Novolin R; Novo Nordisk, Bagsvaerd Denmark) and long-acting insulin analogs (Levemir and Tresiba; Novo Nordisk). Additionally, an insulin pump can be used for continuous subcutaneous insulin delivery as an alternative therapeutic strategy. ${ }^{28}$ To achieve target glycemic control issues associated with insulin therapy, a continuous subcutaneous insulin infusion (CSII) pump (Medtronic MiniMed 620G Insulin pump; Medtronic Inc, Dublin, Ireland) was used for one Pdx1-Hes $1 \mathrm{Tg}$ pig. Because the pump weighed approximately $100 \mathrm{~g}$, we used short-acting and long-acting insulin until the pig reached 7 weeks of age and then used an insulin pump thereafter. 


\section{Biochemical analysis}

Blood samples were collected from the jugular or ear vein. Blood glucose levels were measured using a glucose test meter (GLUCOCARD GT-1820; Arkray Inc, Kyoto, Japan) every day from after birth to 36 weeks after birth. Blood biochemistry for 24 biochemical parameters was measured at several points (FUJI DRI-CHEM 7000; FUJIFILM Corporation, Tokyo, Japan). The concentration of insulin was determined using the enzyme method (measured by SRL, Inc, Tokyo, Japan).

\section{Histology}

At 1 day and 5, 9, 12, 16, and 36 weeks after birth, pigs were sacrificed and subjected to routine necropsy. Selected organs were weighed. The retina, liver, pancreas, and kidney were cut in half, and paraffin-embedded and frozen samples were prepared. For one half of each specimen, the tissue sample was immediately fixed in $4 \%$ paraformaldehyde, embedded in paraffin, and sectioned at $4 \mu \mathrm{m}$ thickness. Cryosections were also prepared and sectioned at $8 \mu \mathrm{m}$ thickness. Previously described immunohistochemical methods were used. ${ }^{29}{ }^{30}$ Briefly, after deparaffinization and blocking, the sections were incubated with diluted primary antibodies overnight at $4^{\circ} \mathrm{C}$.

For the pancreas, the following primary antibodies were used: goat anti-Pdx-1 (Abcam plc, Cambridge, UK), guinea pig anti-insulin (LifeSpan BioSciences, Inc, Washington, USA), mouse antiglucagon (Sigma Aldrich Japan, Tokyo, Japan), antisomatostatin (Sigma Aldrich Japan), and antiamylase (Abcam plc). The secondary antibodies included Alexa488-conjugated, Alexa594-conjugated, and Alexa568-conjugated antibodies (Abcam plc). Nuclei were counterstained with Vectashield mounting medium (Vector Laboratories, Burlingame, California, USA) or Hoechst 33 342. The negative controls included sections that were incubated only with the secondary antibodies. For the retina, isolectin B4 (Thermo Fisher Scientific, Waltham, Massachusetts, USA) was used to visualize blood vessels and microglia. For the liver, Oil-red O staining was carried out using a frozen section. For the kidney, periodic acid-Schiff, periodic acid-methenamine-silver, and Masson-Trichrome staining were performed to examine the production of glycogen or fibers. ${ }^{31}$ The sections were examined under a confocal microscope (BZ-X700; Keyence, Osaka, Japan), and software was used for the data analysis (BZ-H3A; Keyence).

\section{RNA isolation, complementary DNA (cDNA) synthesis, and quantitative real-time PCR (qPCR)}

Gene expression profiles were determined using qPCR, which was performed using a StepOne Plus Real-Time PCR System (Thermo Fisher Scientific) with Premix Ex Taq (Probe qPCR) (Takara Bio), according to the manufacturer's instructions. The TaqMan probes and primer sets are listed in online supplemental table S1. Total RNA was isolated from the pancreas using an RNeasy Plus Mini Kit (Qiagen) and was digested using RNase-free DNaseI (Thermo Fisher Scientific). First-strand cDNA was synthesized using SuperScript III First-Strand Synthesis SuperMix (Thermo Fisher Scientific) and oligo $(\mathrm{dT})_{20}$. The $\Delta \Delta$ CT-method was used to determine the relative expression of genes after normalization to $\beta$-actin $(A C T B)$ expression. The data were collected from at least three independent experiments.

\section{Statistical analyses}

Data were averaged and expressed as means (SD). To compare groups, the unpaired Student's t-tests, repeatedmeasures one-way analysis of variance, and Fisher's protected least significant difference tests were used. Results with $\mathrm{p}$ values of less than 0.05 compared with the WT were considered statistically significant.

\section{RESULTS}

\section{Production of Tg pigs}

We obtained 59 piglets from five litters as offspring of the Pdx1-Hes1 Tg-chimeric boar. These litters included 30 Pdx1-Hes 1 Tg piglets (50.8\%) that showed pancreatic agenesis. Some of the piglets were confirmed by Southern blot analysis to carry 15-20 copies of the transgene (figure 1B), indicating that multicopy transgenes were transmitted from the Tg-chimeric boar to the offspring. The integration site of the Pdx1-Hes 1 transgenes was confirmed by FISH (figure 1C) at chromosome $7 \mathrm{q} 21$. Thus, these findings showed that single-site integration of the transgenes was faithfully inherited from the Tg-chimeric boar to the offspring. All Tg piglets exhibited elevated blood glucose levels.

The WT pig pancreas has three lobes, that is, the duodenal (corresponding to the head of the pancreas), connective (ventral, corresponding to the uncinate process), and splenic (dorsal, corresponding to the body and tail in the human pancreas) lobes. Between the connective and splenic lobes, there is a bridge serving as an anatomical connection between the splenic and connecting lobes. ${ }^{31}$ At 5 weeks after birth, the duodenal and splenic lobes of $\mathrm{Tg}$ pigs were morphologically elongated and narrow, whereas the connective lobe and bridge were absent, indicating pancreatic agenesis (figure 1D, left panel).

\section{$\mathrm{Tg}$ pancreas and physiological features of the $\mathrm{Tg}$ pigs}

Pdx1-Hes $1 \mathrm{Tg}$ pigs without any treatment (TG group; figure $1 \mathrm{E}$, green line) were dead within 8 days after birth (median time to death: 5.5 days). The Pdx1-Hes 1 $\mathrm{Tg}$ pigs with insulin injection treatment (Tg group) exhibited an improved clinical state, indicating that insulin was responsible for survival (figure 1E, blue line). The Tg group still exhibited deficiencies in the connective lobe and bridge of the pancreas (figure 1F,G). Bodyweight increased, consistent with the developmental changes in both the Tg and WT groups (figure $1 \mathrm{H}$ ). However, relative body weight was markedly reduced, reaching $60 \%$ that of WT pigs at 9 weeks of age (Tg: $20.0 \pm 2.6$ vs WT: $33.6 \pm 2.7 \mathrm{~kg}$; $\mathrm{p}=0.01$ ) and $43 \%$ that of WT pigs at 16 weeks of age (Tg: 

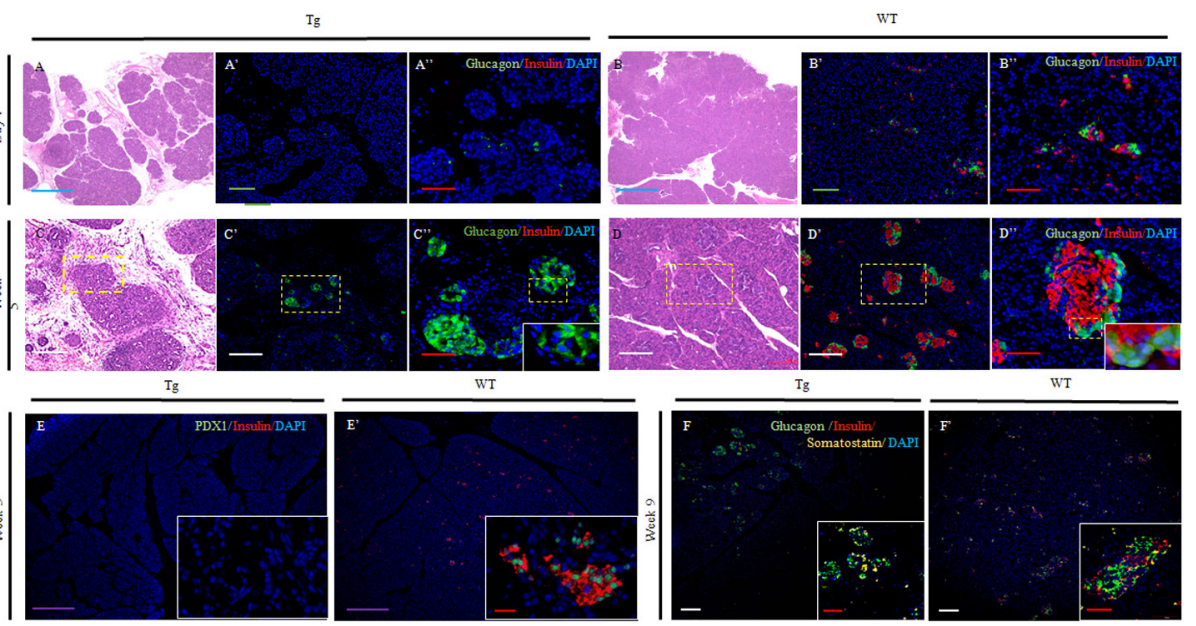

Figure 2 Comparison of protein expression associated with islets in the splenic lobe. (A-D) H\&E staining of the pancreas at day 1 and 5 weeks. Day 1 (A: Tg; B: WT). HE staining showed loose, dispersed, larger shapes in Tg pigs (A) than in WT pigs (B). Week 5 (C: Tg; D: WT). Lobular structures in Tg pigs were present in the pancreas but were thin. Thickening was observed as a consequence of connective tissue surrounding the lobular structures (C). (A'-D', A"-D") Fluorescence micrographs showing glucagon-positive cells. Glucagon-positive cells (A'-D': cytoplasm; green) and merged images (A"-D": insulin-positive cells, cytoplasm; red). The nuclei are stained blue with DAPI. Day 1 (A', A": Tg; B, B": WT). Week 5 (C', C": Tg; D, D": WT). (E, E') Staining of the transcription factor Pdx1 and insulin. Fluorescence micrographs showing Pdx1 (nuclear; green) and insulin staining (cytoplasm; red) in Tg (E) and WT pigs (E'). The nuclei are stained blue with DAPI. (F, F') Staining of islet markers, glucagon, somatostatin, and insulin at 9 weeks. Fluorescence micrographs showing the expression of glucagon (cytoplasm; green), somatostatin (cytoplasm; yellow), and insulin (cytoplasm; red). The nuclei are stained blue with DAPI. Exocrine and endocrine cells throughout the glands. (G, H and G', H') Amylase and insulin staining. Fluorescence micrographs show the expression of amylase (white) and insulin (red). The nuclei are stained blue with DAPI. Day 1 (G: Tg; G': WT). Week 5 (H: Tg, H': WT). (I, I') Triple staining for amylase, glucagon, and insulin at 5 weeks. Fluorescence micrographs showing the expression of glucagon (cytoplasm; green), insulin (cytoplasm; gray), and amylase (cytoplasm; red). The nuclei are stained blue with Hoechst 33 342. Scale bars: blue: $1000 \mu \mathrm{m}$; purple: $500 \mu \mathrm{m}$; green: $200 \mu \mathrm{m}$; white: $100 \mu \mathrm{m}$; red: $50 \mu \mathrm{m}$. Time after birth is indicated as days and weeks. DAPI, 4',6-diamidino-2-phenylindole; Tg, transgenic pigs; WT, wild-type pigs.

$26.7 \pm 2.5$ vs WT: $62 \mathrm{~kg}$ ). The pancreas in $\mathrm{Tg}$ pigs was small (online supplemental table S2; $<<0.001$ ). Pigs with CSII showed improved survival (online supplemental figure S1B), although there were some problems with the CSII pump, including dislocation of the needle and pump failure (online supplemental figure S1C). The body and pancreatic weights of pigs in the $\mathrm{Tg}$ group were lower than those in the WT group (online supplemental table S3).

\section{Morphologies in endocrine cells in the splenic lobe}

In the $\mathrm{Tg}$ group, loose connective tissue was dotted with lobular structures in the parenchyma (figure 2A) on day 1 after birth. These changes were enhanced from head to tail in the splenic lobe. Glucagon-positive cells were scattered throughout the glands; however, these cells were scarce (figure 2A', $\mathrm{A}^{\prime \prime}$ ). At 5 weeks after birth, the connective tissues surrounding the lobular structures were markedly thickened (figure 2C). Glucagon-positive cells were arranged in small aggregates, although the cytoarchitecture remained immature (figure $2 \mathrm{C}^{\prime \prime}$ ). No insulin-positive cells were detected at all stages (figure 2A",C" and online supplemental figure $\left.1 \mathrm{D}, \mathrm{D}^{\prime}\right)$.

In the WT group, islet cell clusters (ICCs) were detected on day 1, and some ICCs consisted only of insulin-positive cells, whereas others contained both glucagon-positive and insulin-positive cells. Glucagon-positive cells were located within the cores and at the peripheries of the islets (figure 2B',B"). At 5 weeks after birth, well-defined small islets had formed (figure 2D',D").

Triple immunostaining for somatostatin, glucagon, and insulin was carried out to confirm whether islet structures in the $\mathrm{Tg}$ group expressed other endocrine markers. The islet structures were positive for both glucagon and somatostatin but negative for insulin at all stages (figure 2F). Moreover, these cells did not exhibit Pdx1 expression (figure 2E), and there were no apparent differences in amylase staining in either group at all stages (figure 2G-I'). The expression patterns of insulin and glucagon were the same between the duodenal and splenic lobes (data not shown).

\section{Quantitative serological analyses}

In the $\mathrm{Tg}$ group, blood glucose levels for offspring exhibited significant elevations ( $\mathrm{Tg}, 816.3 \pm 45$ vs $\mathrm{WT}$, $109.7 \pm 11.3 \mathrm{mg} / \mathrm{dL}$; online supplemental table S4). At 36 weeks, blood glucose levels ranged from $382.0 \pm 56.3$ to $517.0 \pm 49.6 \mathrm{mg} / \mathrm{dL}$ in the $\mathrm{Tg}$ group (WT: $102.5 \pm 6.4$ to $125.3 \pm 16.3 \mathrm{mg} / \mathrm{dL}$; figures $1 \mathrm{I}$ and $3 \mathrm{E}$ and online supplemental table $\mathrm{S} 4$ ). Insulin production was not detected in the Tg group at any time point, indicating that functional $\beta$ cells did not exist (figure $2 \mathrm{~A}^{\prime \prime}, \mathrm{C}^{\prime \prime}, \mathrm{E}-\mathrm{I}$, online supplemental figure 1D' and online supplemental tables S4 and S5). Amylase levels in both groups 

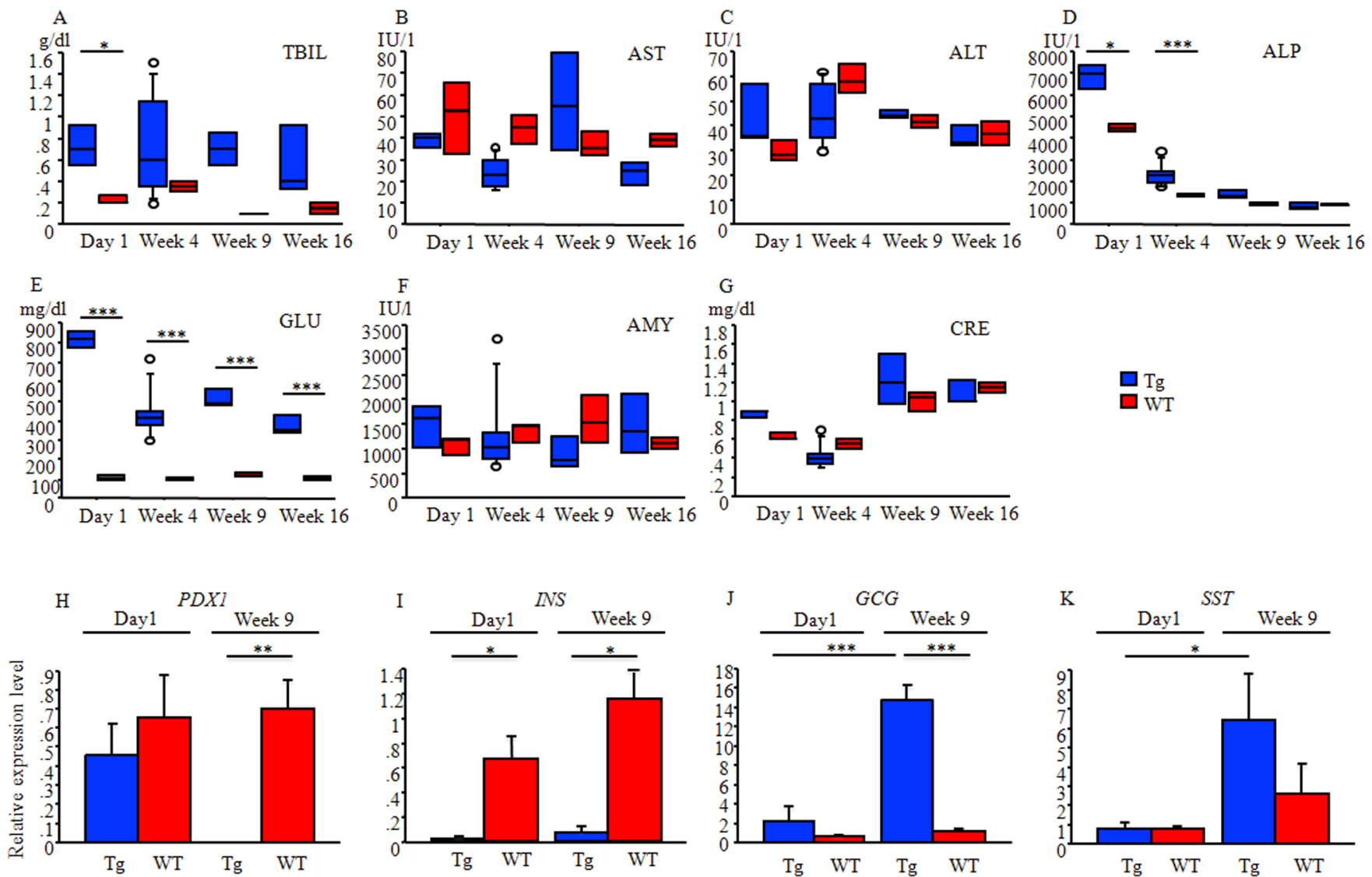

Figure 3 Biochemical parameters and comparison of mRNA levels of genes associated with islets. Biochemical parameters (A) TBIL, (B) AST, (C) ALT, (D) ALP, (E) GLU, (F) AMYL and (G) CRE. Tg: 1 day and 4, 8, 9, and 16 weeks: $n=3$, 8, 7, 3, and 3, respectively. WT: 1 day and 9 and 16 weeks: $n=3,8,7,3$, and 3, respectively. Comparison of mRNA levels of genes associated with islets. $(\mathrm{H}-\mathrm{K})$ mRNA levels of the following genes were detected using qPCR: $(\mathrm{H})$ pancreatic and duodenal homeobox 1 (Pdx1), (I) insulin (INS), (J) glucagon (GCG), and (K) somatostatin (SST). The mRNA levels were normalized to the expression of $\beta$-actin $(A C T B)$ and presented as fold change relative to the WT pancreas each day after birth. Time after birth is indicated as days and weeks. The expression levels were determined based on averages from triplicate assays. Quantitative data are presented as means \pm SDs. ${ }^{*} \mathrm{P}<0.05,{ }^{\star *} \mathrm{p}<0.01,{ }^{\star \star \star} \mathrm{p}<0.001$ versus WT. ALP, alkaline phosphatase; ALT, alanine aminotransferase; AMYL, amylase; AST, aspartate aminotransferase; CRE, creatinine; GLU, glucose; qPCR, quantitative real-time PCR; TBL, total bilirubin; Tg, transgenic pigs; WT, wild-type pigs.

were similar at all time points (Tg: $922.0 \pm 348.8$ to $1491.3 \pm 658.7$ vs WT: $1114.0 \pm 110.0$ to $1609.8 \pm 593.7 \mathrm{mg} /$ $\mathrm{dL}$ ), and the functions of exocrine cells in the Tg group were normal (figure $3 \mathrm{~F}$ and online supplemental tables S4 and S5).

\section{Gene expression profiles associated with islets}

In the TG group, $\mathrm{Pdx} 1$ expression was confirmed 1 day after birth, and the expression level was comparable with that in the WT group; however, Pdx1 expression was not detected at 9 weeks after birth $(p<0.01$; figure $3 \mathrm{H})$. Insulin expression was barely detected at both time points (day 1 ; week $9, \mathrm{p}<0.05$; figure $3 \mathrm{I}$ ). Moreover, glucagon expression in the Tg group was markedly higher at 9 weeks after birth than that in the WT group $(\mathrm{p}<0.001)$ and was more abundant at 9 weeks after birth than at 1 day after birth $(p<0.0001$; figure 3J). Finally, somatostatin level was more abundant at 9 weeks after birth than at 1 day after birth $(\mathrm{p}<0.05$; figure $3 \mathrm{~K})$.

\section{Secondary alterations \\ Eyes}

At 5 weeks after birth, white pupils were observed owing to the development of cataracts (figure 4A-D). Cataracts were not detected in $\mathrm{Tg}$ pigs with CSII (online supplemental figure $\mathrm{S} 1 \mathrm{~F}$ ).

To investigate early changes in the retina in Tg pigs, we performed immunohistochemical analysis of the retina (figure 4G-H"). At the surface of the retina, there were more microglia in Tg pigs than in WT pigs. Microglia showed an amoeboid shape in the retina of Tg pigs in contrast with the WT retina, where microglia showed a relatively ramified shape.

\section{Liver}

Because the biliary epithelium is associated with pancreatic development, ${ }^{24}{ }^{32}$ we next evaluated liver morphology. In the TG group, there were no abnormalities in intrahepatic bile ducts (figure 4I,J). Liver specimens were positive for Oil-red $\mathrm{O}$ staining from 9 weeks 


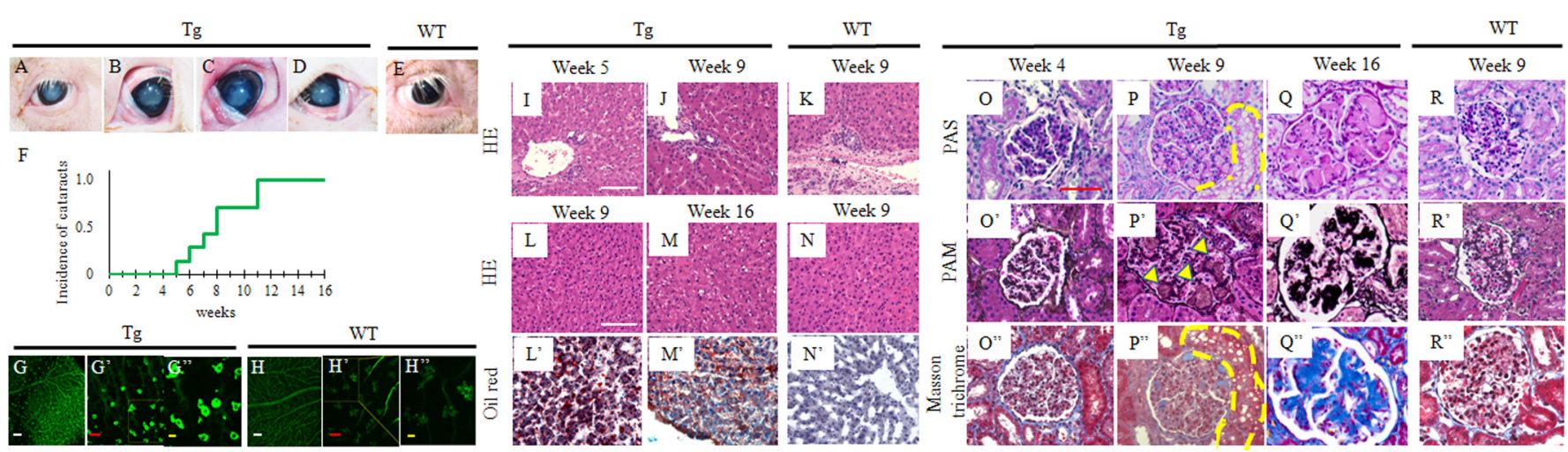

Figure 4 Secondary alterations. Secondary alterations in the eyes. (A-E) Cataracts. The lenses from 5 weeks showed progressive cataracts with increasing opacity in Tg pigs (A-D), whereas those of WT pigs remained clear (E). (A-D) Tg. (A) 5 weeks, (B) 6 weeks, (C) 7 weeks, and (D) 9 weeks. (E) WT: 9 weeks. (F) Incidence of cataracts. (G, G" and H, H”) Isolectin B4-immunofluorescence labeling of the retina (G -G": Tg; H -H": WT). Scale bars: white: $100 \mu \mathrm{m}$; red: $30 \mu \mathrm{m}$; yellow: $10 \mu \mathrm{m}$. Secondary alterations in the liver. (I-K) Intrahepatic bile ducts. H\&E staining. There were no abnormalities in intrahepatic bile ducts in Tg pigs (I and J). (I and J) Tg. (I) 5 weeks, (J) 9 weeks. (K) WT: 5 weeks. (L-N) Hepatic parenchyma. H\&E staining (L and M: Tg; N: WT). (L) 9 weeks, (M) 16 weeks. (N) 9 weeks. (L'-N') Oil-red O staining. Scale bars: $100 \mu$ m. Secondary alterations in the kidneys. (O-R) Periodic acid-Schiff staining, (O'-R') periodic acid-methenamine-silver, and (O"-R") Masson-Trichrome staining. (O-Q, O'-Q', O"-Q") Tg. (O-O”) 4 weeks, (P-P”) 9 weeks, (Q-Q") 16 weeks. (R-R”) WT: 9 weeks. Armanni-Ebstein-like lesions were detected at 9 weeks in Tg pigs (yellow dotted line: P, (P”). Nodular lesions, as indicated with yellow arrows, were observed in Tg pigs but not WT pigs. Nodular lesions with mesangial matrix accumulations were detected in the renal glomeruli of Tg pigs and increased over time (O'-Q', O"-Q"). Scale bar=50 $\mu \mathrm{m}$. Time after birth is indicated as days and weeks. Tg, transgenic pigs; WT, wild-type pigs.

after birth (figure $4 \mathrm{~L}^{\prime}, \mathrm{M}^{\prime}$ ), indicating hepatic steatosis. ${ }^{33}$ These changes were not observed in the WT group (figure $4 \mathrm{~N}, \mathrm{~N}^{\prime}$ and online supplemental figure $1 \mathrm{G}^{\prime}, \mathrm{H}^{\prime}$ ). Aspartate aminotransferase and alanine aminotransferase levels in the Tg group were comparable with those in the WT group and remained within the normal range until sacrifice (figure 3B,C; online supplemental table S4). The biliary enzymes, total bilirubin (TBIL), and alkaline phosphatase (ALP) levels were significantly higher in the Tg group (TBIL: 1 day and 9 weeks; ALP: 1 day and 4 and 9 weeks; figure 3A,D and online supplemental table $\mathrm{S} 4 ; \mathrm{p}<0.01, \mathrm{p}<0.001$ vs WT). In Tg pigs with CSII, Oilred $\mathrm{O}$ staining was negative (online supplemental figure $\mathrm{S} 1 \mathrm{H}$ ), and there were no increases in some of the above parameters (online supplemental table S5).

\section{Kidneys}

Nodular lesions with mesangial matrix accumulation were detected in the renal glomeruli in the Tg group and increased over time (figure 4O'-Q' $)$. In the Tg group, the rate of the lesions (nodular lesion positive glomeruli/ total number of glomeruli per slide) was elevated (Tg; 4 weeks: $4.5 \%, \mathrm{n}=1 ; 9$ weeks: $25.7 \% \pm 16.1 \%, \mathrm{n}=3 ; 16$ weeks: $74 \% \pm 11.6 \%, \mathrm{n}=3)$. At 36 weeks, the rate was the same as on week four for $\mathrm{Tg}$ pigs ( $\mathrm{Tg}: 5 \%, \mathrm{n}=1$ ). Insulin therapy of a CSII pump might have been effective in preventing the progression of renal damage.

Armanni-Ebstein-like lesions ${ }^{33}$ were also detected along the renal glomeruli in the $\mathrm{Tg}$ group and increased over time (yellow dotted line; figure $4 \mathrm{P}, \mathrm{P}^{\prime \prime}$ ). In the Tg group, the rate of the lesions (Armanni-Ebstein-like lesion positive glomeruli/total number of glomeruli per slide) was increased (Tg: 4 weeks: $0 \%, \mathrm{n}=1 ; 9$ weeks: $8.5 \% \pm 4.1 \%$, $\mathrm{n}=3 ; 16$ weeks: $21.6 \% \pm 3.4 \%, \mathrm{n}=3)$. These changes were also confirmed in Tg pigs with CSII at 36 weeks, although the rate was only $1.1 \%$ (online supplemental figure S1I-K). These lesions were not identified in WT pigs (figure $4 R^{\prime}, R^{\prime \prime}$ ).

Creatinine levels were within the normal range (online supplemental table S4), and urine samples showed no significant differences in any values in the comparison of $\mathrm{Tg}$ and WT groups (online supplemental table S6). In Tg pigs with CSII, changes in serological values related to the kidney were less pronounced (online supplemental tables $\mathrm{S} 5$ and $\mathrm{S} 7$ ).

\section{DISCUSSION}

Pdx1-Hes1 Tg pigs exhibit several unique properties, including severe diabetes, pancreatic agenesis, abnormal islet structure, no functional or morphological changes in exocrine cells of the biliary tract, and diabetes-associated pathological alterations.

Pdx1 expression controls pancreatic development, ${ }^{11} 12$ 15-17 34 and high expression of Pdx1 facilitates the development of proper pancreas specification and outgrowth from the foregut endoderm. ${ }^{35}$ In acinar cells, low $P d x 1$ expression is required for their formation and differentiation. ${ }^{36}$ Tanihara et al generated $P d x 1$ modified pigs using the CRISPR/Cas9 system introduced into zygotes and investigated the mosaicism, phenotypes, and inheritance of the resulting pigs. Pigs with high mutation rates $(67.7 \%$ and $79.7 \%)$ exhibited hypoplasia of the pancreas. In contrast, pigs with low mutation rates were healthy. ${ }^{37}$ In our preliminary trial, we realized that $20-30$ copies of the Pdx1-Hes1 transgene in midgestational 
fetuses resulted in anomalies in organs, including anal atresia. As shown by our qPCR results, endogenous expression of $P d x 1$ on day one after birth resulted in a $30 \%$ reduction in expression compared with that in the WT group. Then, Pdx1 expression was suppressed, which may have blocked insulin gene transcription. Thus, our data showed that a certain level of endogenous Pdx1 expression was necessary for the extension of life span in the Tg group, despite the occurrence of hyperglycemia.

In contrast, transduction with the Pdx1-Hes 1 transgene did not influence the production of glucagon or somatostatin.

Studies in mice have provided insights into the presence of the duodenal and splenic lobe, but the absence of the connective lobe of the pancreas in Tg pigs. In Pdx1-null mutants, the dorsal pancreas initially develops via budding, and the bud undergoes limited branching outgrowth. Ventral bud formation is not maintained as a discrete structure. ${ }^{1134}$ Thus, the duodenal and splenic lobes of the pancreas may develop, even in the presence of low $P d x 1$ expression; however, the connective lobe likely requires an adequate level of $P d x 1$ expression. Accordingly, there may be differences in spatial development between the dorsal and ventral pancreas, and specific timing and signals are likely needed for the proper development of the pancreas.

Agenesis of the pancreas is a rare anatomical abnormality of the human pancreas. Schwitzgebel et $a l^{88}$ demonstrated that mutations in the insulin promoter factor 1 gene was one of the causes leading to pancreatic agenesis. In this case, an infant was born at term from nonconsanguineous parents and presented with intrauterine growth retardation. At the age of 12 days, hyperglycemia was observed, and neonatal diabetes was diagnosed. The infant was treated with an insulin pump. Despite euglycemia, the infant did not gain weight, and exocrine pancreas insufficiency was diagnosed. Imaging studies, including abdominal ultrasound and CT scan, revealed no pancreas. In another case, agenesis of the dorsal lobe of pancreas (ADP) was reported. ADP patients have no specific symptoms, and such patients can be identified through imaging examinations for common abdominal symptoms such as pain or bloating. At present, the pathogenesis of this disease is not fully understood. ${ }^{39}$ In our $\mathrm{Tg}$ model with genetically engineered pigs, the symptoms emerged drastically, but the dorsal lobe was present and the exocrine pancreas was normal. Thus, the pattern for emergence of pancreatic agenesis was different from humans.

In this study, we chose the Hes1 gene to generate $\mathrm{Tg}$ pigs for several reasons. First, Hes1 has critical roles during exocrine pancreatic development. Moreover, Hes1 suppresses endocrinal development through the Notch signal pathway. During pancreas development, the onset of exocrine differentiation is driven by the activity of the pancreas transcription factor (PTF1) transcriptional complex, which is composed of the class II bHLH protein Ptfla and a class I E-box binding partner. ${ }^{40} 41$
Hes1 directly interacts with Ptf1-p48 and binds to Ptf1p48 to downregulate the activity of the PTF1 transcriptional complex, enabling Notch effector proteins to delay exocrine pancreatic differentiation. Hes1 also functions as a general negative regulator of endodermal endocrine differentiation by directly suppressing the expression of the endocrine progenitor gene $N g n 3 .^{24}{ }^{42}$ During endoderm development, endocrine cells exhibit atonal and achaete/scute-related bHLH protein expression. These proteins are antagonized by the Notch pathway, partly acting through HES proteins. ${ }^{23}$ Thus, Hes1 has critical roles during pancreatic exocrine development and endocrine cell regulation. Additionally, Hes1 also mediates biliary organogenesis by preventing pancreatic differentiation. ${ }^{24}$ The interactions between Pdx1 and Hes1 regulate cholangiocyte proliferation in response to injury. ${ }^{32}$ Thus, biliary epithelium and pancreatic development are closely related, and we explored biliary epithelial cells by histological analysis and evaluation of hepatobiliary enzymes. Notably, Tg pigs showed suppression of pancreas and $\beta$ cell development, but the hepatobiliary system was not affected.

In our study, $\mathrm{Tg}$ pigs showed perinatal death owing to severe diabetes. We first attempted glucose control in neonatal and juvenile pigs; however, this approach was extremely difficult to implement. The pigs showed symptoms consistent with the brittle type of diabetes. ${ }^{43}$ CSII may offer an alternative therapeutic strategy in this case. ${ }^{28}$ However, the CSII pump was too heavy for use in neonatal pigs, and the use of the CSII pump attached to the piglets' cage (with the insertion of an insulin injection needle using a long tube) was also ineffective because the tubes became clogged, and the needles were detached. One pig in this study was placed on a CSII pump, although the pump could only be used after the pig grew large enough.

This study focused on pig survival and characterization of Tg pigs using a minimum dose of insulin. Insufficient insulin therapy reportedly stunts growth in diabetic children. ${ }^{44}$ Thus, in future studies, it will be necessary to evaluate the appropriate dose of insulin to control blood glucose levels in these piglets.

Secondary alterations due to the complications of diabetes cause vision loss and renal and neurological dysfunctions. The eyes are vulnerable to diabetes and, consequently, develop diabetic retinopathy and cataracts. In fact, diabetic retinopathy is a major cause of blindness among the working population worldwide. Cataracts account for more than half of all cases of blindness, regardless of age. Our immunohistochemical analysis confirmed microglial activation in the retinas of $\mathrm{Tg}$ pigs. A previous study also demonstrated that microglia were activated as early as 4 weeks after the onset of diabetes in a murine model of streptozotocin-induced type 1 diabetes. ${ }^{45}$ Additionally, chronic inflammation has been shown to play crucial roles in the development of diabetic retinopathy. ${ }^{46}$ Although we did not implement biomicroscopic examination to detect typical signs of retinopathy, 
our model may have exhibited the development of clinically significant retinopathy as diabetes persists. Kleinwort $e t a l^{47}$ investigated the incidence of retinopathy in another diabetic pig model-the INSC94Y Tg pigs. We will apply their methodology for the investigation of retinopathy in $\mathrm{Tg}$ pigs to investigate the development of retinopathy in a future study.

Diabetic nephropathy is another disease that leads to end-stage kidney failure. ${ }^{148}$ One histological characteristic of human diabetic nephropathy is the presence of nodular lesions in the glomeruli, that is, nodule-like structures that are formed by mesangial matrix expansion. The number of glomeruli includes nodular lesions. In addition, Armanni-Ebstein lesions have been mostly described in the kidney of patients with poorly controlled diabetes. ${ }^{33}$ The rate of these lesions increased with time in $\mathrm{Tg}$ pigs underlying the worsening of diabetes in $\mathrm{Tg}$ pigs. Renal dysfunction was not evidenced by biochemical analysis; however, the lesions identified in $\mathrm{Tg}$ pigs may occur at the onset of diabetes-associated complications. These changes were milder in Tg pigs with CSII. Reproduction of diabetes-associated complications similar to the clinical course of diabetes in humans is challenging in large animal models ${ }^{6}$; however, we have shown that our model may be useful for elucidating the cause of and developing novel treatments for diabetic nephropathy. ${ }^{31}$

In conclusion, we generated Pdxl-Hes $1 \mathrm{Tg}$ pigs showing induction of a stable diabetic phenotype and diabetes-associated complications relatively early. This model is expected to have applications in determining the roles of Pdx1-Hes 1 during embryogenesis. Additional studies using this model are needed to identify the timing of Pdx1 suppression and to assess the reasons for the upregulation of glucagon and somatostatin in the TG group. We also plan to evaluate whether changes in Pdx1-Hes 1 transgene copy-numbers can affect the severity of diabetes and whether structural changes in peripheral nerves of $\mathrm{Tg}$ pigs could develop with increasing age. Although the transcription factor cascades that control the pancreas and pancreatic endocrine cell differentiation and function are well investigated in rodents, ${ }^{8911142324}$ they remain largely unknown in pigs. Very recently, Kim et $a t^{49}$ demonstrated that both pig and human $\beta$ and $\alpha$ cells share characteristic molecular and developmental features by showing stage-specific gene expression patterns that are not observed in mice. These findings show how studies of pig pancreas and pancreatic islet may complement our understanding of the developmental programs relevant to human pancreatic diseases. The Tg pigs would be useful to investigate the role of $P d x 1$ and/or Hes 1 for pancreatic development in large animal models.

Finally, we are now conducting $\beta$ cell replacement therapies with human-derived insulin-producing cells in $\mathrm{Tg}$ pigs. ${ }^{50}$ The model is also useful for elucidating the underlying causes and the development of novel treatments for diabetic retinopathy and nephropathy, as well as the complications of diabetes.
Author affiliations

${ }^{1}$ Meiji University International Institute for Bio-Resource Research, Meiji University - Ikuta Campus, Kawasaki, Japan

${ }^{2}$ Department of Immunology, St. Marianna University School of Medicine, Kawasaki, Japan

${ }^{3}$ Laboratory of Medical Bioengineering, Department of Life Sciences, School of Agriculture, Meiji University, Kawasaki, Japan

${ }^{4}$ Center for iPS Cell Research and Application (CiRA), Kyoto University, Kyoto, Japan ${ }^{5}$ Division of Ophthalmology, Department of Visual Sciences, Nihon University School of Medicine, Tokyo, Japan

${ }^{6}$ Department of Organ Fabrication, Keio University, School of Medicine, Tokyo, Japan

${ }^{7}$ Division of Stem Cell Therapy, Institute of Medical Science, Institute of Medical Science, The University of Tokyo, Tokyo, Japan

${ }^{8}$ Institute for Stem Cell Biology and Regenerative Medicine, Department of Genetics, Stanford University School of Medicine, Stanford, California, USA

Contributors MN and HirosN conception and design, financial support, collection and assembly of data, data analysis and interpretation, manuscript writing and final approval of manuscript. KH, MW, KN, KaO, TY, AU, HM and KU collection and assembly of data, data analysis and interpretation. HY and TN collection and assembly of data, data analysis and interpretation. $\mathrm{Ke} 0$, EK and HiromN data analysis and interpretation.

Funding This research work was supported by Grants-in-Aid from the Ministry of Education, Culture, Sports, Science, and Technology of Japan (17K10527 and $20 \mathrm{~K} 08944$ to MN), the Japan Agency for Medical Research and Development (Leading Advanced Projects for Medical Innovation, Generation of Functional Organs using Developmental Niche (to HirosN), Japan Science and Technology Agency (Exploratory Research for Advanced Technology, NAKAUCHI Stem Cell and Organ Regeneration (to HirosN), and the Meiji University International Institute for Bio-Resource Research (to $\mathrm{MN}$ and HirosN).

Disclaimer The funding organizations had no roles in the study's design, data collection and analysis, the decision to publish, or the manuscript's preparation.

Competing interests None declared.

Patient consent for publication Not required.

Ethics approval All animal experiments in this study were approved by Meiji University's Institutional Animal Care and Use Committee (IACUC07-0005, 14-0008, 19-0003).

Provenance and peer review Not commissioned; externally peer reviewed.

Data availability statement All data relevant to the study are included in the article or uploaded as supplemental information.

Supplemental material This content has been supplied by the author(s). It has not been vetted by BMJ Publishing Group Limited (BMJ) and may not have been peer-reviewed. Any opinions or recommendations discussed are solely those of the author(s) and are not endorsed by BMJ. BMJ disclaims all liability and responsibility arising from any reliance placed on the content. Where the content includes any translated material, BMJ does not warrant the accuracy and reliability of the translations (including but not limited to local regulations, clinical guidelines, terminology, drug names and drug dosages), and is not responsible for any error and/or omissions arising from translation and adaptation or otherwise.

Open access This is an open access article distributed in accordance with the Creative Commons Attribution Non Commercial (CC BY-NC 4.0) license, which permits others to distribute, remix, adapt, build upon this work non-commercially, and license their derivative works on different terms, provided the original work is properly cited, appropriate credit is given, any changes made indicated, and the use is non-commercial. See: http://creativecommons.org/licenses/by-nc/4.0/.

ORCID ID

Masaki Nagaya http://orcid.org/0000-0003-0784-5009

\section{REFERENCES}

1 Cole JB, Florez JC. Genetics of diabetes mellitus and diabetes complications. Nat Rev Nephrol 2020;16:377-90.

2 Shapiro AMJ, Pokrywczynska M, Ricordi C. Clinical pancreatic islet transplantation. Nat Rev Endocrinol 2017;13:268-77.

3 Russ HA, Parent AV, Ringler JJ, et al. Controlled induction of human pancreatic progenitors produces functional beta-like cells in vitro. Embo J 2015;34:1759-72. 
4 Veres A, Faust AL, Bushnell $\mathrm{HL}$, et al. Charting cellular identity during human in vitro $\beta$-cell differentiation. Nature 2019;569:368-73.

5 Zhou Q, Melton DA. Pancreas regeneration. Nature 2018;557:351-8.

6 Umeyama K, Watanabe M, Saito H, et al. Dominant-Negative mutant hepatocyte nuclear factor 1 alpha induces diabetes in transgeniccloned pigs. Transgenic Res 2009;18:697-706.

7 Renner S, Braun-Reichhart C, Blutke A, et al. Permanent neonatal diabetes in INS(C94Y) transgenic pigs. Diabetes 2013;62:1505-11.

8 Kageyama R, Ohtsuka T, Tomita K. The bHLH gene Hes1 regulates differentiation of multiple cell types. Mol Cells 2000;10:1-7.

9 Bastidas-Ponce A, Scheibner K, Lickert $\mathrm{H}$, et al. Cellular and molecular mechanisms coordinating pancreas development. Development 2017;144:2873-88.

10 Jennings RE, Berry AA, Strutt JP, et al. Human pancreas development. Development 2015;142:3126-37.

11 Jonsson J, Carlsson L, Edlund T, et al. Insulin-promoter-factor 1 is required for pancreas development in mice. Nature 1994;371:606-9.

12 Stoffers DA, Zinkin NT, Stanojevic V, et al. Pancreatic agenesis attributable to a single nucleotide deletion in the human IPF1 gene coding sequence. Nat Genet 1997;15:106-10.

13 Waeber G, Thompson N, Nicod P, et al. Transcriptional activation of the GLUT2 gene by the IPF-1/STF-1/IDX-1 homeobox factor. Mol Endocrinol 1996;10:1327-34.

14 Holland AM, Góñez LJ, Naselli G, et al. Conditional expression demonstrates the role of the homeodomain transcription factor Pdx in maintenance and regeneration of beta-cells in the adult pancreas. Diabetes 2005;54:2586-95.

15 Jennings RE, Berry AA, Kirkwood-Wilson R, et al. Development of the human pancreas from foregut to endocrine commitment. Diabetes 2013;62:3514-22.

16 Thomas IH, Saini NK, Adhikari A, et al. Neonatal diabetes mellitus with pancreatic agenesis in an infant with homozygous IPF-1 Pro63fsX60 mutation. Pediatr Diabetes 2009;10:492-6.

17 Nicolino M, Claiborn KC, Senée V, et al. A novel hypomorphic PDX1 mutation responsible for permanent neonatal diabetes with subclinical exocrine deficiency. Diabetes 2010;59:733-40.

18 Lee JE. Basic helix-loop-helix genes in neural development. Curr Opin Neurobiol 1997;7:13-20.

19 Sasai Y, Kageyama R, Tagawa Y, et al. Two mammalian helix-loophelix factors structurally related to Drosophila hairy and enhancer of split. Genes Dev 1992;6:2620-34.

20 Apelqvist A, Li H, Sommer L, et al. Notch signalling controls pancreatic cell differentiation. Nature 1999;400:877-81.

21 Murtaugh LC, Stanger BZ, Kwan KM, et al. Notch signaling controls multiple steps of pancreatic differentiation. Proc Natl Acad Sci U S A 2003;100:14920-5.

22 Esni F, Ghosh B, Biankin AV, et al. Notch inhibits PTF1 function and acinar cell differentiation in developing mouse and zebrafish pancreas. Development 2004;131:4213-24.

23 Jensen J, Pedersen EE, Galante P, et al. Control of endodermal endocrine development by HES-1. Nat Genet 2000;24:36-44.

24 Sumazaki R, Shiojiri N, Isoyama S, et al. Conversion of biliary system to pancreatic tissue in Hes1-deficient mice. Nat Genet 2004;36:83-7.

25 Matsunari $\mathrm{H}$, Nagashima $\mathrm{H}$, Watanabe $\mathrm{M}$, et al. Blastocyst complementation generates exogenic pancreas in vivo in apancreatic cloned pigs. Proc Natl Acad Sci U S A 2013;110:4557-62.

26 Kapellen TM, Heidtmann B, Lilienthal E, et al. Continuous subcutaneous insulin infusion in neonates and infants below 1 year: analysis of initial bolus and basal rate based on the experiences from the German Working group for pediatric pump treatment. Diabetes Technol Ther 2015;17:872-9.

27 Secrest AM, Becker DJ, Kelsey SF, et al. Characterizing sudden death and dead-in-bed syndrome in type 1 diabetes: analysis from two childhood-onset type 1 diabetes registries. Diabet Med 2011;28:293-300

28 Nevo-Shenker M, Phillip M, Nimri R, et al. Type 1 diabetes mellitus management in young children: implementation of current technologies. Pediatr Res 2020;87:624-9.
29 Nagaya M, Katsumata Y, Arai Y, et al. Effectiveness of bioengineered islet cell sheets for the treatment of diabetes mellitus. J Surg Res 2018;227:119-29.

30 Nagaya M, Hayashi A, Nakano K, et al. Distributions of endocrine cell clusters during porcine pancreatic development. PLoS One 2019;14:e0216254.

31 Umeyama K, Nakajima M, Yokoo T, et al. Diabetic phenotype of transgenic pigs introduced by dominant-negative mutant hepatocyte nuclear factor $1 \alpha$. J Diabetes Complications 2017;31:796-803.

32 Marzioni M, Saccomanno S, Agostinelli L, et al. PDX-1/Hes-1 interactions determine cholangiocyte proliferative response to injury in rodents: possible implications for sclerosing cholangitis. $J$ Hepatol 2013:58:750-6.

33 Kodikara S, Paranitharan P, Pollanen MS. The role of the ArmanniEbstein lesion, hepatic steatosis, biochemical analysis and second generation anti-psychotic drugs in fatal diabetic ketoacidosis. $J$ Forensic Leg Med 2013;20:108-11.

34 Offield MF, Jetton TL, Labosky PA, et al. Pdx-1 is required for pancreatic outgrowth and differentiation of the rostral duodenum. Development 1996;122:983-95.

35 Fujitani Y, Fujitani S, Boyer DF, et al. Targeted deletion of a cisregulatory region reveals differential gene dosage requirements for $\mathrm{Pdx} 1$ in foregut organ differentiation and pancreas formation. Genes Dev 2006;20:253-66.

36 Hale MA, Kagami H, Shi L, et al. The homeodomain protein PDX1 is required at mid-pancreatic development for the formation of the exocrine pancreas. Dev Biol 2005;286:225-37.

37 Tanihara F, Hirata M, Thi Nguyen N, et al. Generation of viable PDX1 gene-edited founder pigs as providers of nonmosaics. Mol Reprod Dev 2020;87:471-81.

38 Schwitzgebel VM, Mamin A, Brun T, et al. Agenesis of human pancreas due to decreased half-life of insulin promoter factor $1 . J$ Clin Endocrinol Metab 2003;88:4398-406.

39 Mei W, Cao F, Li F. Two cases of agenesis of the dorsal pancreas and a review of the literature. BMC Gastroenterol 2020;20:94.

40 Yoshitomi H, Zaret KS. Endothelial cell interactions initiate dorsal pancreas development by selectively inducing the transcription factor Ptf1a. Development 2004;131:807-17.

41 Ghosh B, Leach SD. Interactions between hairy/enhancer of Splitrelated proteins and the pancreatic transcription factor PTF1-P48 modulate function of the PTF1 transcriptional complex. Biochem $J$ 2006;393:679-85.

42 Jørgensen MC, de Lichtenberg KH, Collin CA, et al. Neurog3dependent pancreas dysgenesis causes ectopic pancreas in Hes 1 mutant mice. Development 2018;145. doi:10.1242/dev.163568. [Epub ahead of print: 03 Sep 2018].

43 Garrett CJ, Choudhary P, Amiel SA, et al. Recurrent diabetic ketoacidosis and a brief history of brittle diabetes research: contemporary and past evidence in diabetic ketoacidosis research including mortality, mental health and prevention. Diabet Med 2019;36:1329-35.

44 Tattersall RB, Pyke DA. Growth in diabetic children. Studies in identical twins. Lancet 1973;2:1105-9.

45 Krady JK, Basu A, Allen CM, et al. Minocycline reduces proinflammatory cytokine expression, microglial activation, and caspase-3 activation in a rodent model of diabetic retinopathy. Diabetes 2005;54:1559-65.

46 Rübsam A, Parikh S, Fort P. Role of inflammation in diabetic retinopathy. Int J Mol Sci 2018;19:942.

47 Kleinwort KJH, Amann B, Hauck SM, et al. Retinopathy with central oedema in an INS ${ }^{\mathrm{C} 94 \mathrm{Y}}$ transgenic pig model of long-term diabetes. Diabetologia 2017;60:1541-9.

48 Betz B, Conway BR. An update on the use of animal models in diabetic nephropathy research. Curr Diab Rep 2016;16:18.

$49 \mathrm{Kim} \mathrm{S}$, Whitener RL, Peiris $\mathrm{H}$, et al. Molecular and genetic regulation of pig pancreatic islet cell development. Development 2020;147:dev186213.

50 Kieffer TJ, Woltjen K, Osafune K, et al. Beta-cell replacement strategies for diabetes. J Diabetes Investig 2018;9:457-63. 